\title{
OPEN Impacts of lipid-related metabolites, adiposity, and genetic background on blood eosinophil counts: the Nagahama study
}

\author{
Kenta Nishi ${ }^{1}$, Hisako Matsumoto ${ }^{1 凶}$, Noriyuki Tashima ${ }^{1}$, Satoru Terada ${ }^{1}$, Natsuko Nomura ${ }^{1}$, \\ Mariko Kogo ${ }^{1}$, Chie Morimoto ${ }^{1}$, Hironobu Sunadome ${ }^{2}$, Tadao Nagasaki ${ }^{2}$, Tsuyoshi Oguma ${ }^{1}$, \\ Yoshinari Nakatsuka², Kimihiko Murase ${ }^{2}$, Takahisa Kawaguchi ${ }^{3}$, Yasuharu Tabara ${ }^{3}$, \\ Kazuhiro Sonomura ${ }^{3}$, Fumihiko Matsuda ${ }^{3}$, Kazuo $\mathrm{Chin}^{2}$ \& Toyohiro Hirai $^{1}$
}

Blood eosinophil count is a useful measure in asthma or COPD management. Recent epidemiological studies revealed that body mass index (BMI) is positively associated with eosinophil counts. However, few studies focused on the role of adiposity and fatty acid-related metabolites on eosinophil counts, including the effect of genetic polymorphism. In this community-based study involving 8265 participants (30-74 year old) from Nagahama city, we investigated the relationship between eosinophil counts and serum levels of fatty acid-related metabolites. The role of $M D C 1$, a gene that is related to eosinophil counts in our previous study and encodes a protein that is thought to be involved in the repair of deoxyribonucleic acid damage, was also examined taking into account its interaction with adiposity. Serum levels of linoleic acid (LA) and $\beta$-hydroxybutyric acid (BHB) were negatively associated with eosinophil counts after adjustment with various confounders; however, there were positive interactions between serum LA and BMI and between serum BHB and BMI/body fat percentages in terms of eosinophil counts. In never-smokers, there was positive interaction for eosinophil counts between the CC genotype of MDC1 rs4713354 and BMI/body fat percentages. In conclusion, both serum LA and BHB have negative impacts on eosinophil counts, while adiposity shows robust positive effects on eosinophil counts, partly via genetic background in never-smokers.

Peripheral blood eosinophil counts are one of the important clinical indices in the management of asthma and $\mathrm{COPD}^{1-4}$, and several epidemiological studies have been conducted to clarify factors affecting blood eosinophil counts. Eosinophil has also attracted special interest for its role in preventing obesity by Beijing of white adipose tissue in mouse models ${ }^{5,6}$. We and other research groups showed that higher body mass index (BMI), in addition to other factors such as allergic inflammation, male population, smoking history, and genetic backgrounds, is associated with elevated blood eosinophil counts ${ }^{7-11}$. As there were various reports in different cohorts that showed the association of blood eosinophil counts with factors contributing to metabolic syndrome, including lipoproteins or triglyceride $(\mathrm{TG})^{10,12-14}$, the potential role of adiposity in blood eosinophil counts had been suggested. However, few studies have focused on the role of adiposity and metabolites in lipid metabolism on blood eosinophil counts ${ }^{12,15}$. In addition, how genetic background contributes to the association between adiposity and blood eosinophil counts remains unknown.

Free fatty acid (FFA), an important source of energy, is an intermediate product of lipid mobilization and is usually released into blood by the breakdown from TG in adipose tissue when energy demand is increased ${ }^{16}$. Therefore, although FFA is often elevated in obesity, the behavior of FFAs with blood eosinophil counts might be different from that of other lipoproteins and TG, yet the interrelation between FFAs and blood eosinophil counts remain unknown. In addition, butyrate, one of the short-chain fatty acids (SCFAs), and an important metabolite in obesity prevention ${ }^{17,18}$, is thought to be protective against type 2 inflammation and induces apoptosis of eosinophils via histone deacetylase (HDAC) inhibitory activity ${ }^{19,20}$. However, only small amounts of

${ }^{1}$ Department of Respiratory Medicine, Kyoto University Graduate School of Medicine, 54 Kawaharacho, Shogoin, Sakyo-ku, Kyoto 606-8507, Japan. ${ }^{2}$ Department of Respiratory Care and Sleep Control Medicine, Kyoto University Graduate School of Medicine, Kyoto, Japan. ${ }^{3}$ Center for Genomic Medicine, Kyoto University Graduate School of Medicine, Kyoto, Japan. ${ }^{\bowtie}$ email: matsumoto.hisako.v92@kyoto-u.jp 


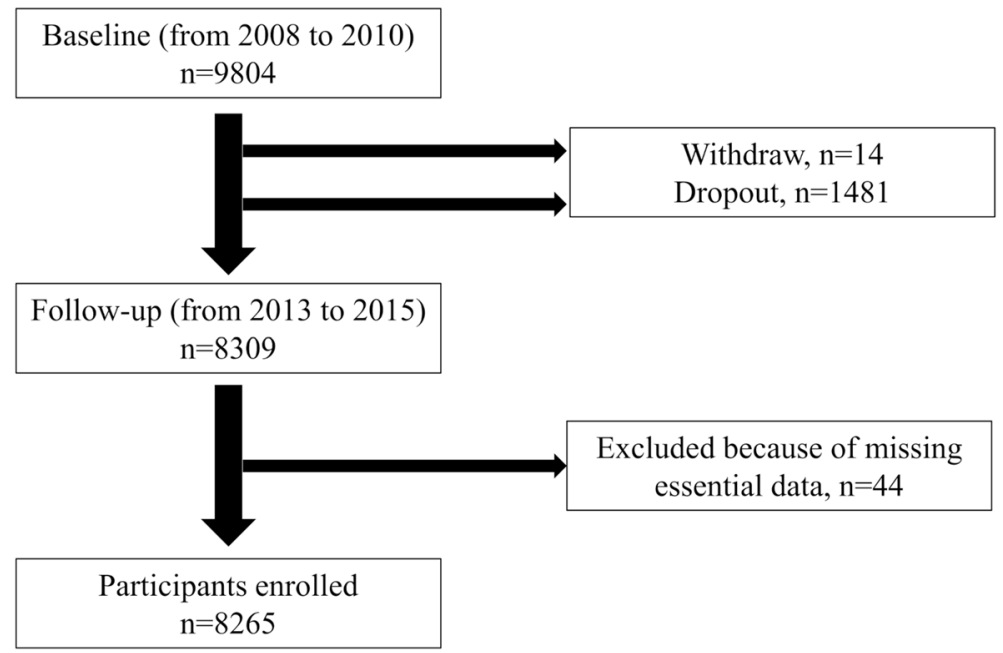

Figure 1. Participants flow diagram. Out of 9804 participants who were surveyed in the baseline assessment, 8265 participants were analyzed in the follow-up assessment.

endogenous butyrate appear in peripheral blood which makes it difficult to be quantified ${ }^{21}$. In contrast, a ketone body $\beta$-hydroxybutyric acid (BHB), which is a derivative of butyrate ${ }^{22}$ and is derived from the $\beta$-oxidation of FFAs in case of glucose deficiency or impaired glucose utilization ${ }^{23}$, is measureable in blood. BHB is a potential candidate that might reduce blood eosinophil counts, owing to the fact that BHB also inhibits HDACs ${ }^{24,25}$.

Genetic backgrounds may have some effects on the association between obesity/BMI and blood eosinophil counts. $M D C 1$ located in $6 \mathrm{p} 21$, of which variant we previously identified as a potential risk gene for elevated blood eosinophil counts via genome-wide association study (GWAS) and human leukocyte antigen (HLA) imputation ${ }^{10}$, is involved in deoxyribonucleic acid (DNA) damage repair, due to its encoding protein which is a mediator of DNA damage checkpoint protein $1^{26}$. We hypothesized that a MDC1 variant might have some effects on the association between obesity and higher blood eosinophil counts, as obesity is one of the inducers of DNA damage ${ }^{27}$.

In this epidemiological study, we comprehensively investigated associations between fatty acid-related metabolites and blood eosinophil counts. Interactions between obesity and genetic backgrounds for blood eosinophil counts were also explored. Should these associations exist, it would contribute largely to understanding the effects of adiposity on blood eosinophil counts.

\section{Results}

A total of 8309 subjects participated in the follow-up study from November 2013 to November 2015. After exclusion of subjects with missing essential data, 8265 subjects were analyzed (Fig. 1). Table 1 shows the characteristics of the participants involved in the follow-up study.

Associations between serum lipid-related metabolites and blood eosinophil counts. In the univariate analysis, higher serum levels of TG and lower HDL-C associated with higher BMI, body fat percentage, and both blood neutrophil and eosinophil counts (Supplementary Table S1). In contrast, serum FFA levels were found to show negative association with blood eosinophil counts, while showing positive association with BMI and blood neutrophil counts (Supplementary Table S1). We then analyzed contributions of metabolites in fatty acid metabolism to lower blood eosinophil counts. First, the partial least-squares discriminant analysis (PLS-DA) was performed to identify fatty acid-related metabolites associated with lower blood eosinophil counts (Fig. 2), which yielded BHB and linoleic acid (LA) with the variable importance in the projection (VIP) score over 1.5. The Pearson's correlation coefficient between serum BHB and LA was $0.65(P<0.0001)$. Lactic acid, which showed a VIP score of over 1.5 for higher blood eosinophil counts, accounted for non-correlation with blood eosinophil counts as observed in the multivariate analysis (standardized partial regression coefficient, -0.010 ; $95 \% \mathrm{CI}-0.033$ to $0.013 ; P=0.40)$.

Clinical characteristics of subjects with higher BHB and LA. Clinical characteristics of subjects with higher serum BHB (Fig. 3a) and LA (Fig. 3b) were females and never-smokers. Serum BHB and LA manifested negative associations with BMI, but both serum BHB and LA levels tended to distribute in $U$ shape against BMI-the lowest in the overweight group (BMI, $25-30 \mathrm{~kg} / \mathrm{m}^{2}$ ) and numerically elevated in the obese population (BMI $\geq 30 \mathrm{~kg} / \mathrm{m}^{2}$ ) (Fig. 3a,b). Serum BHB, but not LA, showed significant positive association with $\% \mathrm{FEV}_{1}$ (standardized partial regression coefficient, $0.021 ; 95 \%$ CI $0.000-0.041 ; P=0.048$ ), after adjustment with age, sex, BMI, smoking history, blood eosinophil counts, elapsed time after a meal, and medication for dyslipidemia. 


\begin{tabular}{|c|c|}
\hline & $n=8265$ \\
\hline Age (years) & $62.0(47.0-70.0)^{\mathrm{a}}$ \\
\hline Sex (male, $n, \%)$ & $2648,32.0 \%$ \\
\hline BMI $\left(\mathrm{kg} / \mathrm{m}^{2}\right)$ & $22.2(3.3)^{\mathrm{b}}$ \\
\hline Body fat percentages (\%) & $25.3(7.5)^{\mathrm{b}}$ \\
\hline Smoking (current/ex/never, \%) & $10.2 / 21.5 / 68.2$ \\
\hline$\% \mathrm{FEV}_{1}(\%)$ & $102.2(16.2)^{\mathrm{b}}$ \\
\hline Blood eosinophils $(/ \mu \mathrm{L})$ & $110.0(68.8-182.9)^{\mathrm{a}}$ \\
\hline Blood neutrophils $(/ \mu \mathrm{L})$ & $2786.4(2242.7-3494.7)^{\mathrm{a}}$ \\
\hline Serum total IgE (IU/mL) & $65.3(27.2-168)^{\mathrm{a}}$ \\
\hline High-sensitivity CRP (ng/mL) & $359(175.0-762.3)^{\mathrm{a}}$ \\
\hline Total cholesterol $(\mathrm{mg} / \mathrm{dL})$ & $203.5(33.7)^{\mathrm{b}}$ \\
\hline LDL cholesterol (mg/dL) & $117.5(29.0)^{\mathrm{b}}$ \\
\hline HDL cholesterol (mg/dL) & $67.3(17.4)^{\mathrm{b}}$ \\
\hline Triglycerides (mg/dL) & $79.0(57.0-111.0)^{\mathrm{a}}$ \\
\hline Free fatty acids $(\mathrm{mEq} / \mathrm{L})$ & $0.58(0.19)^{\mathrm{b}}$ \\
\hline
\end{tabular}

Table 1. Participants' characteristics. BMI body mass index, FEV1 forced expiratory volume in $1 \mathrm{~s} .{ }^{\text {a Median }}$ (interquartile range, IQR). ${ }^{\mathrm{b}}$ Mean (SD).

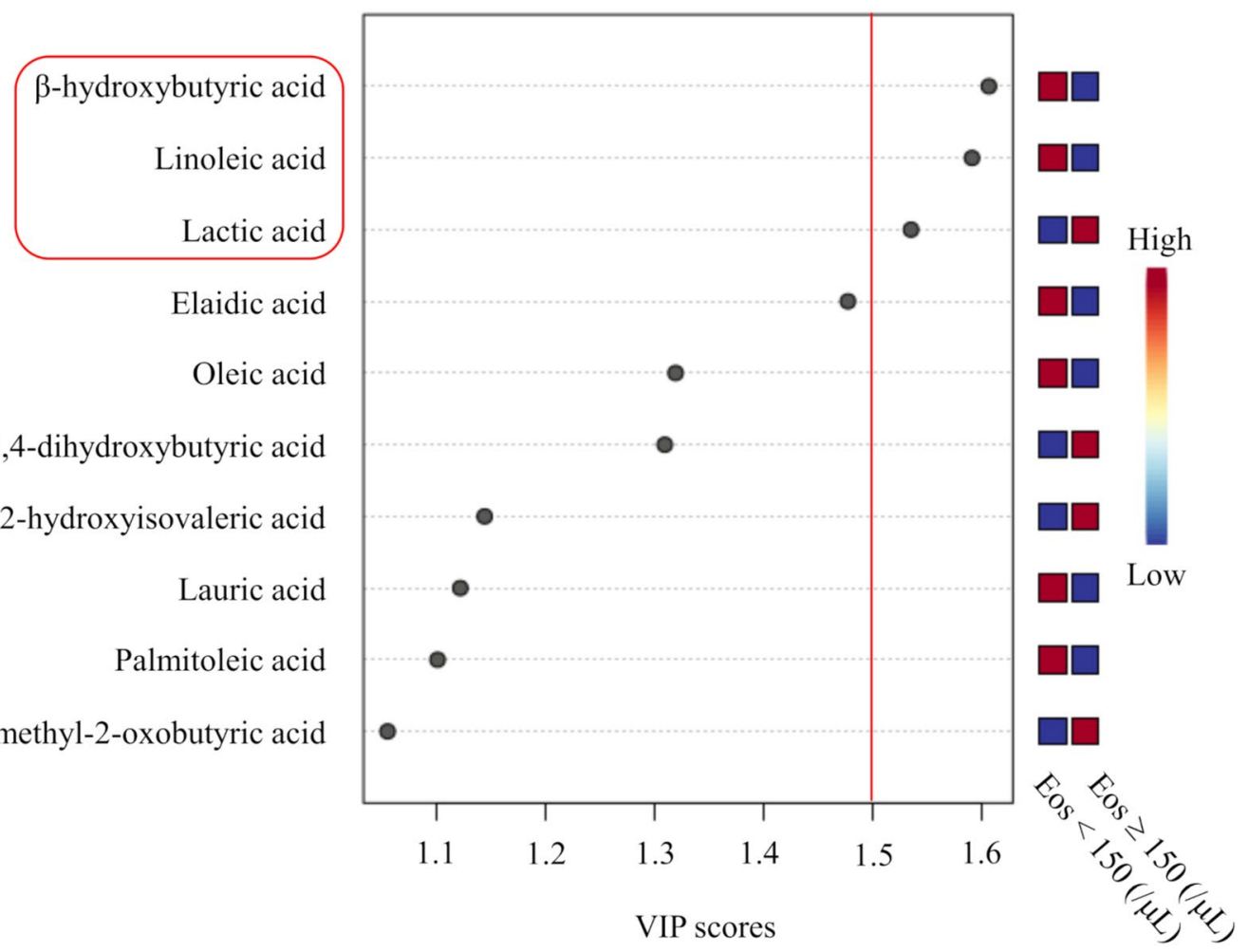

Figure 2. Partial least-squares discriminant analysis (PLS-DA). $\beta$-Hydroxybutyric acid (BHB) and linoleic acid (LA) were screened as candidates associated with lower blood eosinophil counts, with variable importance in projection (VIP) scores $>1.5$. Eos blood eosinophil counts.

Multivariate analysis for associations of BHB, LA, and adiposity with blood eosinophil counts. In the multivariate analysis, higher serum BHB and LA were both significantly associated with lower blood eosinophil counts and higher blood neutrophil counts independent of age, sex, smoking history, elapsed time after a meal, medication for dyslipidemia, serum total IgE, HDL-C, TG, and high-sensitivity CRP (Supplementary Table S2). However, significant positive interactions for blood eosinophil counts between serum BHB and BMI (Model 1 in Table 2, Fig. 4) or body fat percentages (Model 2 in Table 2) and between serum LA and BMI (Model 1 in Table 2) were observed. There were no interactions for eosinophil counts between serum BHB or LA and sex or smoking history. 
A
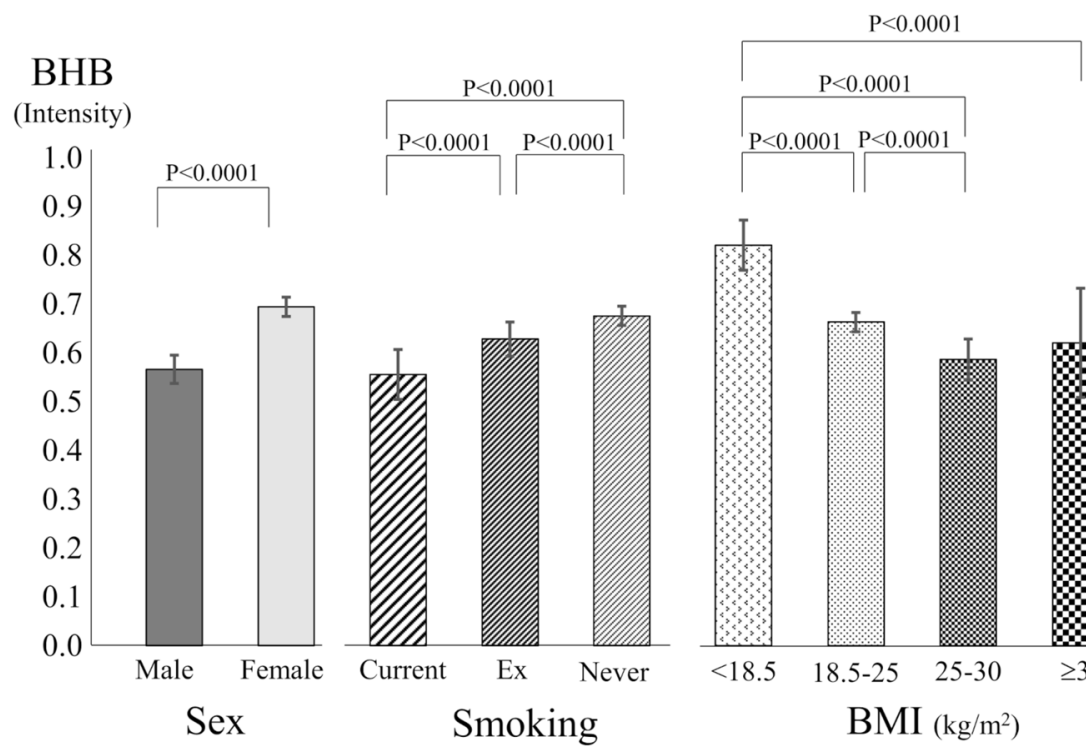

\section{B}

\section{LA}

(Intensity)

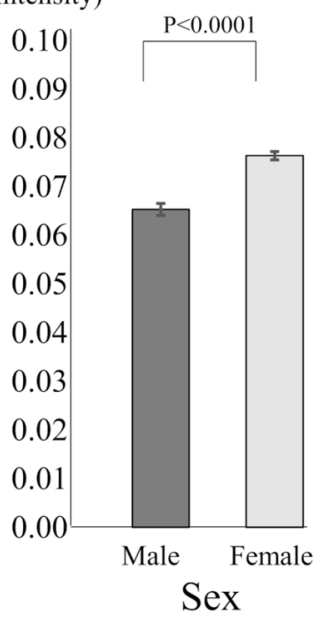

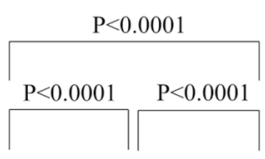

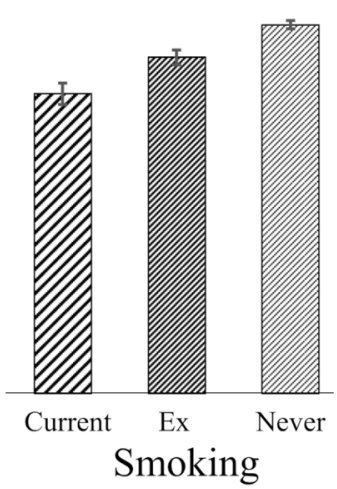

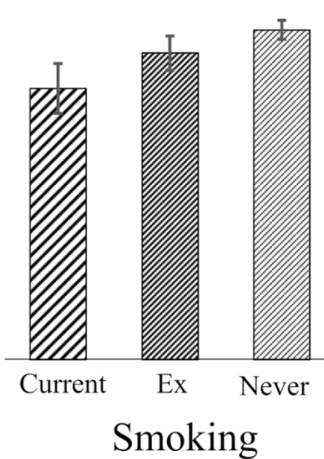

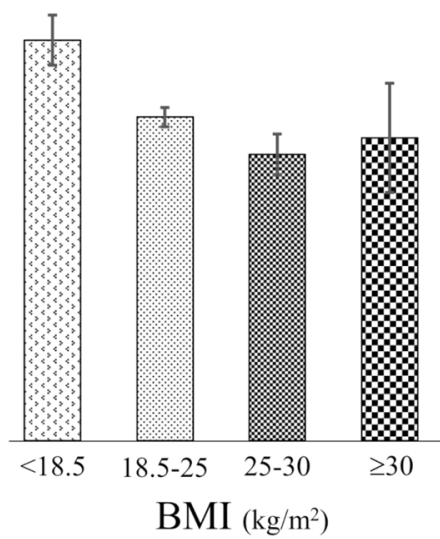

Figure 3. Serum levels of (a) BHB and (b) LA according to sex, smoking status, or body mass index (BMI) levels: underweight, $18.5>\mathrm{BMI}\left(\mathrm{kg} / \mathrm{m}^{2}\right)$; normal, $25>\mathrm{BMI} \geq 18.5\left(\mathrm{~kg} / \mathrm{m}^{2}\right)$; overweight, $30>\mathrm{BMI} \geq 25(\mathrm{~kg} /$ $\left.\mathrm{m}^{2}\right)$; obese, BMI $\geq 30\left(\mathrm{~kg} / \mathrm{m}^{2}\right)$. Log-transformed serum BHB and LA were used for the analysis. Means $(95 \%$ confidence interval) are presented.

Impact of genetic background and adiposity on blood eosinophil counts. Finally, we examined genetic effects of CC genotype of rs4713354 ( $M D C 1$ on chromosome 6p21) which was previously identified as a potential risk factor for higher blood eosinophil counts by GWAS and HLA imputation ${ }^{10}$, focusing on its interaction with BMI or body fat percentages for elevated blood eosinophil counts. In the 4177 participants whom we were able to perform GWAS and HLA imputation on, there was a trend toward positive interaction between CC rs4713354 and body fat percentages for elevated blood eosinophil counts. When the analysis was confined to never-smokers ( $n=2840$; AA, AC, CC: 1771, 927, 142, resp.) to exclude the influences of smoking on elevated eosinophil counts, positive interaction for blood eosinophil counts between CC genotype and BMI (Model 1 in Table 3) or body fat percentages (Model 2 in Table 3) was significant. Analysis using LA instead of BHB yielded similar results (Table 4 ). 


\begin{tabular}{|c|c|c|c|c|c|c|}
\hline & \multicolumn{3}{|c|}{ Blood eosinophil counts ${ }^{a}$} & \multicolumn{3}{|c|}{ Blood neutrophil counts ${ }^{a}$} \\
\hline & $\beta$ coefficient & $95 \% \mathrm{CI}$ & $p$ value & $\beta$ coefficient & $95 \%$ CI & $p$ value \\
\hline \multicolumn{7}{|l|}{ Model 1} \\
\hline $\mathrm{BHB}^{\mathrm{a}}$ & -0.074 & -0.096 to -0.052 & $<0.0001$ & 0.162 & $0.141-0.183$ & $<0.0001$ \\
\hline BMI $\left(\mathrm{kg} / \mathrm{m}^{2}\right)$ & 0.067 & $0.042-0.091$ & $<0.0001$ & 0.025 & $0.002-0.048$ & 0.034 \\
\hline $\mathrm{BHB}^{\mathrm{a} * \mathrm{BMI}}$ & 0.028 & $0.007-0.049$ & 0.009 & -0.017 & -0.037 to 0.003 & 0.099 \\
\hline $\mathrm{LA}^{\mathrm{a}}$ & -0.068 & -0.090 to -0.045 & $<0.0001$ & 0.159 & $0.138-0.180$ & $<0.0001$ \\
\hline BMI $\left(\mathrm{kg} / \mathrm{m}^{2}\right)$ & 0.069 & $0.045-0.093$ & $<0.0001$ & 0.018 & -0.005 to 0.041 & 0.12 \\
\hline $\mathrm{LA}^{\mathrm{a} \star} \mathrm{BMI}$ & 0.028 & $0.007-0.049$ & 0.009 & -0.032 & -0.052 to -0.012 & 0.002 \\
\hline \multicolumn{7}{|l|}{ Model 2} \\
\hline $\mathrm{BHB}^{\mathrm{a}}$ & -0.077 & -0.099 to -0.055 & $<0.0001$ & 0.161 & $0.140-0.182$ & $<0.0001$ \\
\hline Body fat percentages (\%) & 0.079 & $0.053-0.106$ & $<0.0001$ & 0.056 & $0.031-0.082$ & $<0.0001$ \\
\hline $\mathrm{BHB}^{\mathrm{a} * \text { Body fat }}$ & 0.028 & $0.007-0.049$ & 0.010 & -0.008 & -0.028 to 0.012 & 0.45 \\
\hline $\mathrm{LA}^{\mathrm{a}}$ & -0.072 & -0.094 to -0.049 & $<0.0001$ & 0.154 & $0.132-0.175$ & $<0.0001$ \\
\hline Body fat percentages (\%) & 0.085 & $0.058-0.112$ & $<0.0001$ & 0.046 & $0.021-0.072$ & 0.0004 \\
\hline $\mathrm{LA}^{\mathrm{a} \star}$ Body fat & 0.020 & -0.001 to 0.041 & 0.066 & -0.024 & -0.044 to -0.004 & 0.019 \\
\hline
\end{tabular}

Table 2. Multivariate analysis for associations between serum fatty acid-related metabolites and blood eosinophil counts or neutrophil counts. $B H B \beta$-hydroxybutyric acid, $L A$ linoleic acid, $B M I$ body mass index, $\beta$ coefficient standardized partial regression coefficient, $C I$ confidence interval. ${ }^{a}$ Log-transformed. Multivariate analysis for blood eosinophil counts were adjusted by age, sex, smoking history, elapsed time after a meal, medication for dyslipidemia, serum HDL cholesterol, serum triglycerides, serum total IgE and high-sensitivity CRP. Multivariate analysis for blood neutrophil counts were adjusted by age, sex, smoking history, elapsed time after a meal, medication for dyslipidemia, serum HDL cholesterol, serum triglycerides and high-sensitivity CRP.

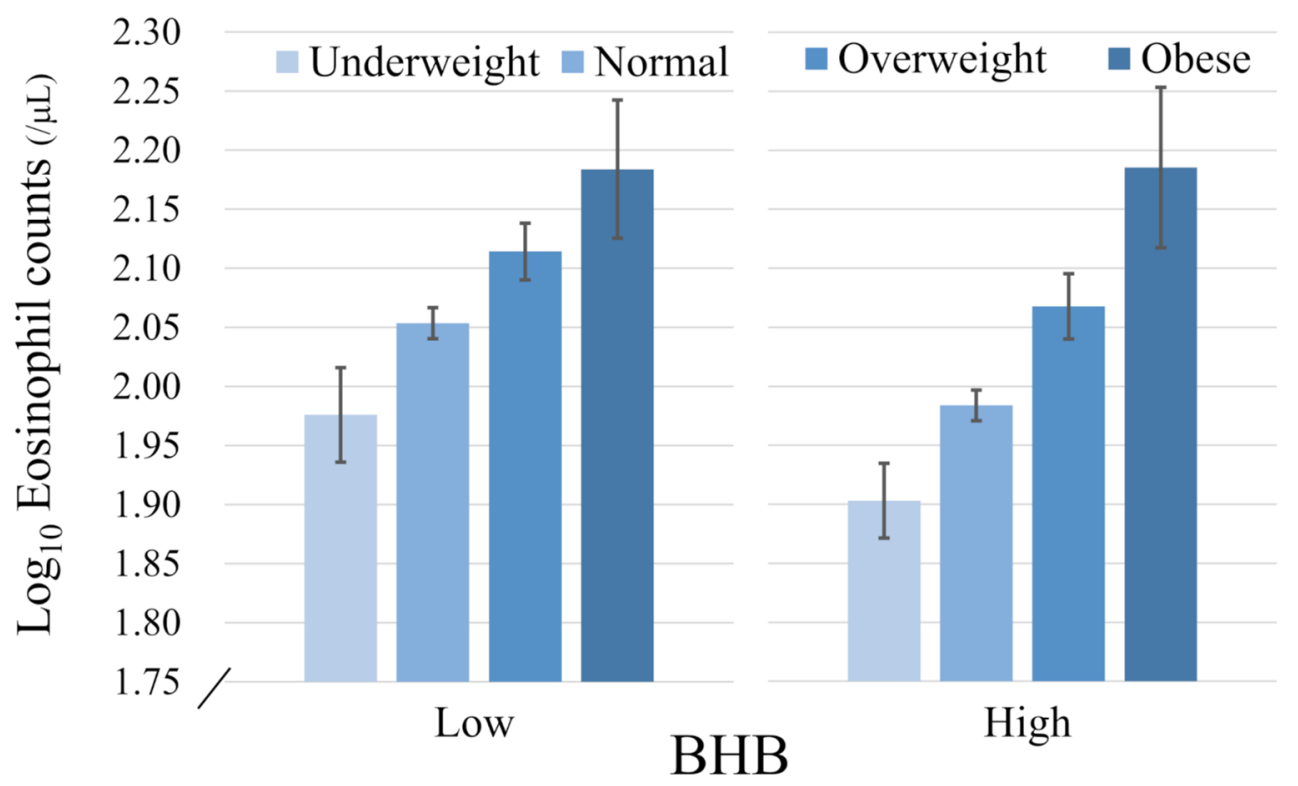

(Intensity)

Figure 4. Association of blood eosinophil counts with serum BHB levels and BMI levels: underweight, $18.5>\mathrm{BMI}\left(\mathrm{kg} / \mathrm{m}^{2}\right)$; normal, $25>\mathrm{BMI} \geq 18.5\left(\mathrm{~kg} / \mathrm{m}^{2}\right)$; overweight, $30>\mathrm{BMI} \geq 25\left(\mathrm{~kg} / \mathrm{m}^{2}\right)$; obese, BMI $\geq 30$ $\left(\mathrm{kg} / \mathrm{m}^{2}\right)$. Subjects with log-transformed $\mathrm{BHB}<$ median were categorized as "low BHB" and those with logtransformed $\mathrm{BHB} \geq$ median were categorized as "high BHB." Log-transformed blood eosinophil counts and serum BHB levels were used for the analysis. Means (95\% confidence interval) are presented. 


\begin{tabular}{|c|c|c|c|}
\hline & $\beta$ coefficient & $95 \% \mathrm{CI}$ & p value \\
\hline \multicolumn{4}{|l|}{ Model 1} \\
\hline $\mathrm{BHB}^{\mathrm{a}}$ & -0.068 & -0.107 to -0.030 & 0.0005 \\
\hline $\mathrm{BMI}\left(\mathrm{kg} / \mathrm{m}^{2}\right)$ & 0.107 & $0.057-0.157$ & $<0.0001$ \\
\hline rs4713354 AC vs AA & 0.069 & $0.030-0.107$ & 0.0005 \\
\hline CC vs AC & 0.036 & -0.003 to 0.074 & 0.070 \\
\hline $\mathrm{BMI}^{\star}(\mathrm{AC}$ vs AA $)$ & -0.026 & -0.074 to 0.021 & 0.27 \\
\hline $\mathrm{BMI}^{\star}(\mathrm{CC}$ vs AC $)$ & 0.043 & $0.004-0.083$ & 0.032 \\
\hline \multicolumn{4}{|l|}{ Model 2} \\
\hline $\mathrm{BHB}^{\mathrm{a}}$ & -0.071 & -0.109 to -0.032 & 0.0003 \\
\hline Body fat percentages (\%) & 0.126 & $0.073-0.178$ & $<0.0001$ \\
\hline rs4713354 AC vs AA & 0.067 & $0.029-0.106$ & 0.0006 \\
\hline CC vs AC & 0.043 & $0.004-0.082$ & 0.032 \\
\hline Body fat ${ }^{*}$ (AC vs AA) & -0.040 & -0.089 to 0.008 & 0.11 \\
\hline Body fat ${ }^{\star}(\mathrm{CC}$ vs AC) & 0.048 & $0.008-0.088$ & 0.020 \\
\hline
\end{tabular}

Table 3. Multivariate analysis in never-smokers for elevated blood eosinophil counts including BHB, BMI or body fat, rs4713354, and its interactions as variables. $B H B \beta$-hydroxybutyric acid, $B M I$ body mass index, $\beta$ coefficient standardized partial regression coefficient, $C I$ confidence interval. ${ }^{a}$ Log-transformed. Adjusted by age, sex, elapsed time after a meal, medication for dyslipidemia, serum HDL cholesterol, serum triglycerides, serum total IgE and high-sensitivity CRP.

\begin{tabular}{|c|c|c|c|}
\hline & $\beta$ coefficient & 95\% CI & p value \\
\hline \multicolumn{4}{|l|}{ Model 1} \\
\hline $\mathrm{LA}^{\mathrm{a}}$ & -0.062 & -0.101 to -0.023 & 0.0005 \\
\hline BMI $\left(\mathrm{kg} / \mathrm{m}^{2}\right)$ & 0.108 & $0.058-0.158$ & $<0.0001$ \\
\hline rs4713354 AC vs AA & 0.069 & $0.031-0.108$ & 0.0005 \\
\hline CC vs AC & 0.035 & -0.003 to 0.074 & 0.070 \\
\hline $\mathrm{BMI}^{\star}(\mathrm{AC}$ vs AA $)$ & -0.026 & -0.073 to 0.022 & 0.27 \\
\hline $\mathrm{BMI}^{\star}(\mathrm{CC}$ vs $\mathrm{AC})$ & 0.042 & $0.003-0.081$ & 0.032 \\
\hline \multicolumn{4}{|l|}{ Model 2} \\
\hline $\mathrm{LA}^{\mathrm{a}}$ & -0.066 & -0.105 to -0.027 & 0.0003 \\
\hline Body fat percentages (\%) & 0.131 & $0.079-0.184$ & $<0.0001$ \\
\hline rs4713354 AC vs AA & 0.068 & $0.029-0.107$ & 0.0006 \\
\hline $\mathrm{CC}$ vs $\mathrm{AC}$ & 0.043 & $0.004-0.082$ & 0.032 \\
\hline Body fat ${ }^{\star}$ (AC vs AA) & -0.041 & -0.090 to 0.007 & 0.11 \\
\hline Body fat ${ }^{\star}(\mathrm{CC}$ vs AC) & 0.048 & $0.008-0.088$ & 0.020 \\
\hline
\end{tabular}

Table 4. Multivariate analysis in never-smokers for elevated blood eosinophil counts including LA, BMI or body fat, rs4713354, and its interactions as variables. $L A$ linoleic acid, BMI body mass index, $\beta$ coefficient

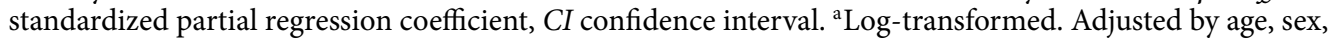
elapsed time after a meal, medication for dyslipidemia, serum HDL cholesterol, serum triglycerides, serum total IgE and high-sensitivity CRP.

\section{Discussion}

This epidemiological study is the first to demonstrate the negative associations of FFAs and its related metabolites, particularly serum levels of LA (n-6 polyunsaturated fatty acid) and BHB (ketone body), with blood eosinophil counts independent of various confounders. In never-smokers, apart from the negative impacts of FFAs on eosinophil counts, there was a positive interaction between body fat percentage and the CC genotype of MDC1 rs4713354 in terms of blood eosinophil counts. This suggested that the subjects with the CC genotype of rs4713354 had a greater increase in blood eosinophil counts related to adiposity than those with AC/AA genotypes (Supplementary Fig. S1).

As shown in previous studies ${ }^{7,12}$, higher body fat percentages, higher serum levels of TG, and lower HDL were associated with elevated blood eosinophil counts and neutrophil counts, which is consistent with the findings in this study. These associations are probably due to chronic systemic inflammation inherent in adiposity ${ }^{28}$, such as leptin and proinflammatory cytokines ${ }^{29}$. In contrast, despite positive associations between serum FFA and BMI or blood neutrophil counts, serum FFA showed negative correlation with blood eosinophil counts. FFA is 
an intermediate product of lipid mobilization, and its serum level is determined by the balance between release from adipose tissue according to energy demand and intake into the liver and adipose tissue when not utilized as an energy source ${ }^{16}$. Therefore, although serum FFA levels are elevated in the obese population, similarly with TG, it is plausible that the association between blood eosinophil counts and FFA is different from that of other lipid metabolites. Indeed, serum LA, a representative long-chain fatty acid that induces systemic inflammation ${ }^{31}$, showed negative association with blood eosinophil counts, despite the fact that LA augments type 2 inflammation in allergic diseases as a source of arachidonic acid when present in excessive quantity ${ }^{30,31}$. The mechanisms underlying the negative association between serum LA and eosinophil counts remain unknown, because an in vitro study shows that LA does not affect eosinophil survival ${ }^{32}$. However, it is possible to speculate that LA may reduce eosinophil counts, when considering that nitrated LA activates peroxisome proliferator-activated receptor gamma $(\mathrm{PPAR} \gamma)$ at nanomolar concentrations ${ }^{33}$, which alleviates type 2 inflammation, although LA in itself is inactive as a PPAR $\gamma$ ligand ${ }^{34}$. In addition, the negative impact of LA on eosinophil counts may reflect that of $\mathrm{BHB}$, when considering that there was a moderate positive correlation between serum LA and BHB that is derived from the $\beta$-oxidation of fatty acids during the period of glucose deficiency.

Furthermore, $\mathrm{BHB}$, which is increased under ketogenic conditions such as starvation or prolonged exercise $\mathrm{e}^{24}$ and therefore is decreased in the obese population, is structurally related to SCFA ${ }^{22}$. As was expected, serum BHB level was negatively associated with blood eosinophil counts. A recent study reported that butyrate, which is mainly produced by the gut microbiota derived from fermentation of dietary fibers ${ }^{22}$, was able to attenuate activity of human eosinophils such as adhesion to the endothelium, migration, and survival. These effects were exerted probably through HDAC inhibition, and not through G protein-coupled receptor (GPR) 41 for SCFAs ${ }^{20}$. Another study reported that SCFA especially butyrate was able to inhibit the proliferation of group 2 innate lymphoid cells (ILC2), a critical player in inducing type 2 inflammation ${ }^{35}$, GATA-binding protein 3 (GATA3) expression, and interleukin-13, interleukin-5 production from ILC2 in mouse models ${ }^{19}$. These effects against allergic inflammation were also considered to occur via HDAC inhibitory activity of butyrate. Since BHB is also known as an HDAC inhibitor, BHB could potentially decrease survival of eosinophils ${ }^{22,24}$.

This epidemiological study confirmed that various factors yield positive impacts on blood eosinophil counts as previously demonstrated ${ }^{7,36}$. For example, although we found negative impacts of serum BHB and LA on blood eosinophil counts, these were attenuated by higher BMI and body fat percentages, as shown by positive interactions for blood eosinophil counts. Additionally, as observed in this study, males and smokers, which are known to be factors for higher blood eosinophil counts in the general population ${ }^{7,10}$, showed lower serum levels of BHB and LA than their counterparts, which is a similar finding of previous studies ${ }^{37-39}$. Thus males, smoking, and lower serum BHB or LA may additively increase blood eosinophil counts.

Genetic backgrounds may also intervene in the robust association between adiposity and elevated blood eosinophil counts. GATA2, IL1RL1, and IL5 are known risk genes for elevated blood eosinophil counts, and proteins encoded by them are well-known factors in type 2 inflammation ${ }^{11}$. Differently from these genes, MDC1 encodes a mediator of DNA damage checkpoint protein 1, which plays an important role in DNA damage response by recruiting a number of repair proteins to the damaged sites ${ }^{26}$. MDC1 rs4713354 which is located in the promoter region of $M D C 1$ is a known functional polymorphism. A to C transversion of rs4713354 causes a loss of transcription factor binding sites, which may lead to the decrease of expression of $M D C 1^{40}$. In the analysis of radiation-induced apoptosis in A549 cells, which are human lung adenocarcinoma-derived epithelial-like cells, apoptosis was largely suppressed with MDC1 small interfering RNA, which was restored with addition of $M D C 1^{41}$. Thus, decreased expression of $M D C 1$ via A to $C$ transversion of rs4713354 may enhance survival of eosinophils that have DNA repair machinery ${ }^{42}$ in the milieu of oxidative DNA damage in obesity ${ }^{27}$. Therefore, we speculated that effects of $M D C 1$ rs4713354 variants may be evident in the obese population. Indeed, there were positive interactions for blood eosinophil counts between CC genotype of rs4713354 and BMI or body fat percentages after excluding the influences of smoking on blood eosinophil counts. These findings suggest that subjects with CC genotype of rs4713354 were susceptible to the effects of adiposity on elevated blood eosinophil counts.

Lastly, apart from eosinophil counts, the association of serum BHB with $\% \mathrm{FEV}_{1}$ is elaborated. Serum $\mathrm{BHB}$, but not LA, correlated with higher $\% \mathrm{FEV}_{1}$ after adjustment with $\mathrm{BMI}$ and other factors, suggesting that $\mathrm{BHB}$ might relax airway smooth muscle. SCFAs like butyrate may cause contraction of airway smooth muscle by activating GPR $41^{43}$. Meanwhile, a study reported that water-soluble BHB acts as antagonist of GPR41 at the level of sympathetic ganglion ${ }^{44}$. When considering the capability of butyrate for contractions of airway smooth muscle, it is possible to speculate that relaxing of airway smooth muscle might be induced by BHB through GPR41 inhibition, although this requires further evidence.

Although butyrate could have strong association with eosinophil apoptosis ${ }^{20}$, neither fecal nor serum butyrate was available in this study, which is one of the considered limitations of this study, since no stool sample was obtained and serum butyrate was difficult to measure. However, serum BHB was examined. Studies focusing on beneficial effects of butyrate and $\mathrm{BHB}$ on eosinophilic inflammation may be prompted by the negative impacts of BHB on blood eosinophil counts observed in this study. Another limitation was the limited investigation of the interactions between BMI and other risk genes for elevated blood eosinophils ${ }^{11}$ : GATA2, IL1RL1, and IL5. In addition, this interaction of MDC1 was not validated in the second population. Nonetheless, the positive interaction for blood eosinophil counts between the variant of $M D C 1$ and BMI/body fat percentages in never-smokers may shed light on the involvement of DNA damage and repair of eosinophils from the perspective of adiposity.

In conclusion, serum FFA and its metabolite, particularly LA and BHB, may decrease blood eosinophil counts, while adiposity increases blood eosinophil counts synergistically with genetic background in never-smokers. These may suggest that the balance of adiposity and its breakdown may affect blood eosinophil counts. 


\section{Methods}

Study design and participants. The Nagahama Prospective Genome Cohort for Comprehensive Human Bioscience (The Nagahama Study) is a community-based prospective cohort study ${ }^{45,46}$. The participants were recruited from generally healthy residents, aged 30-74 years, in Nagahama City, Shiga Prefecture, Japan. A total of 9804 subjects were enrolled from 2008 to 2010, considering the inclusive period the baseline assessment of the Nagahama Study. After 5 years from the baseline assessment, a follow-up survey was conducted from November 2013 to November 2015 on subjects who had not waived their consent. In the present study, subjects were excluded if they had missing essential data of blood eosinophil counts or metabolites. This study obtained approval from the Ethics Committee of Kyoto University Graduate School of Medicine and the Nagahama Municipal Review Board. All methods were performed in accordance with the principles of the Declaration of Helsinki. Written informed consent was obtained from all participants.

Questionnaire and measurements. Clinical measurements were composed of self-reported questionnaires, pulmonary function tests, and blood sampling. Asthma and medication for dyslipidemia was determined by self-reported questionnaire. Next, pulmonary function was measured by a computed spirometer with automated quality checks (SP-350 COPD; Fukuda Denshi, Tokyo, Japan). Aside from serum total IgE, which was measured at baseline assessment only, blood data at follow-up assessment were analyzed. The time elapsed after their last meal was also recorded. Gas chromatography-mass spectrometry (GC-MS) analysis was conducted using GCMS-QP2010 Ultra (Shimadzu, Kyoto, Japan) at follow-up assessment according to the previous study ${ }^{47}$. In brief, water, methanol, chloroform, and the internal standard solution (2-isopropylmalic acid) were added to each serum sample. The supernatant after centrifugation was dried and was processed under trimethylsilyl derivatization. Then, the extract was subjected to GC-MS analysis. For data acquisition and processing, GCMS solution software version 2.71 (Shimadzu, Kyoto, Japan) was used. Metabolites were identified using the Shimadzu GC/MS database or by comparison with authentic standards. The peak area of each metabolite was calculated and normalized.

For genotyping and GWAS, DNA was extracted from blood samples for analysis. The HLA imputation consisted of 4615 variants in total: 3848 single nucleotide polymorphisms (SNPs), 645 HLA amino acids, 53 two-digit HLA alleles, and 69 four-digit HLA alleles. A detailed description of GWAS and HLA imputation is shown in our previous article ${ }^{10}$.

Statistical analysis. PLS-DA was performed using MetaboAnalyst $4.0^{48}$. Subsequent to log-transformation, 24 lipid-related metabolite data (Supplementary Table S3) were mean-centered and divided by the standard deviation of each variable. Then, metabolites with lower blood eosinophil counts were observed using the PLSDA. The other statistical analyses were performed with JMP version Pro15 (SAS institute Inc., Tokyo, Japan). Pearson's correlation coefficient was used to examine correlation between two variables. One-way analysis of variance with post hoc Tukey's Honest Significant Difference test or Student's $t$ test was performed to conduct comparisons in between groups. Next, multivariate regression analyses were performed to identify factors associated with blood eosinophil counts at follow-up assessment. $P$ values of less than 0.05 were regarded as statistically significant.

\section{Data availability}

The data analyzed during the current study are not publicly available because we did not obtain prior consent from the participants, but are available from the corresponding author on reasonable request.

\section{Appendix}

The Nagahama study group executive committee comprises the following individuals: Yasuharu Tabara, Takahisa Kawaguchi, Kazuya Setoh, Yoshimitsu Takahashi, Shinji Kosugi, Takeo Nakayama, and Fumihiko Matsuda from the Center for Genomic Medicine, Kyoto University Graduate School of Medicine (YaT, TK, KS, FM); Department of Health Informatics (YoT, TN); Department of Medical Ethics and Medical Genetics (SK), Kyoto University School of Public Health.

Received: 24 March 2021; Accepted: 13 July 2021

Published online: 28 July 2021

\section{References}

1. Price, D. B. et al. Blood eosinophil count and prospective annual asthma disease burden: A UK cohort study. Lancet Respir. Med. 3, 849-858 (2015).

2. Korevaar, D. A. et al. Diagnostic accuracy of minimally invasive markers for detection of airway eosinophilia in asthma: A systematic review and meta-analysis. Lancet Respir. Med. 3, 290-300 (2015).

3. Roche, N. et al. Blood eosinophils and response to maintenance chronic obstructive pulmonary disease treatment. Data from the FLAME trial. Am. J. Respir. Crit. Care Med. 195, 1189-1197 (2017).

4. Watz, H. et al. Blood eosinophil count and exacerbations in severe chronic obstructive pulmonary disease after withdrawal of inhaled corticosteroids: A post-hoc analysis of the WISDOM trial. Lancet Respir. Med. 4, 390-398 (2016).

5. Knights, A. J. et al. Eosinophil function in adipose tissue is regulated by Krüppel-like factor 3 (KLF3). Nat. Commun. 11, 2922 (2020).

6. Brestoff, J. R. et al. Group 2 innate lymphoid cells promote beiging of white adipose tissue and limit obesity. Nature 519, 242-246 (2015).

7. Hartl, S. et al. Blood eosinophil count in the general population: Typical values and potential confounders. Eur. Respir. J. 55, 1901874 (2020). 
8. Caspard, H., Ambrose, C. S., Tran, T. N., Chipps, B. E. \& Zeiger, R. S. Associations between individual characteristics and blood eosinophil counts in adults with asthma or COPD. J. Allergy Clin. Immunol. Pract. 8, 1606-1613.e1601 (2020).

9. Nagasaki, T. et al. Smoking attenuates the age-related decrease in IgE levels and maintains eosinophilic inflammation. Clin. Exp. Allergy 43, 608-615 (2013).

10. Sunadome, H. et al. Correlation between eosinophil count, its genetic background and body mass index: The Nagahama Study. Allergol. Int. 69, 46-52 (2020).

11. Gudbjartsson, D. F. et al. Sequence variants affecting eosinophil numbers associate with asthma and myocardial infarction. Nat. Genet. 41, 342-347 (2009).

12. Barochia, A. V. et al. High density lipoproteins and type 2 inflammatory biomarkers are negatively correlated in atopic asthmatics. J. Lipid Res. 58, 1713-1721 (2017).

13. Shim, W. S. et al. The association of total and differential white blood cell count with metabolic syndrome in type 2 diabetic patients. Diabetes Res. Clin. Pract. 73, 284-291 (2006).

14. DiSantostefano, R. L., Hinds, D., Le, H. V. \& Barnes, N. C. Relationship between blood eosinophils and clinical characteristics in a cross-sectional study of a US population-based COPD cohort. Respir. Med. 112, 88-96 (2016).

15. Babio, N. et al. White blood cell counts as risk markers of developing metabolic syndrome and its components in the PREDIMED study. PLoS ONE 8, e58354 (2013).

16. Frayn, K. N., Arner, P. \& Yki-Järvinen, H. Fatty acid metabolism in adipose tissue, muscle and liver in health and disease. Essays Biochem. 42, 89-103 (2006).

17. McNabney, S. M. \& Henagan, T. M. Short chain fatty acids in the colon and peripheral tissues: A focus on butyrate, colon cancer, obesity and insulin resistance. Nutrients 9,1348 (2017).

18. Fang, W., Xue, H., Chen, X., Chen, K. \& Ling, W. Supplementation with sodium butyrate modulates the composition of the gut microbiota and ameliorates high-fat diet-induced obesity in mice. J. Nutr. 149, 747-754 (2019).

19. Thio, C. L., Chi, P. Y., Lai, A. C. \& Chang, Y. J. Regulation of type 2 innate lymphoid cell-dependent airway hyperreactivity by butyrate. J. Allergy Clin. Immunol. 142, 1867-1883.e1812 (2018).

20. Theiler, A. et al. Butyrate ameliorates allergic airway inflammation by limiting eosinophil trafficking and survival. J. Allergy Clin. Immunol. 144, 764-776 (2019).

21. Murase, M., Kimura, Y. \& Nagata, Y. Determination of portal short-chain fatty acids in rats fed various dietary fibers by capillary gas chromatography. J. Chromatogr. B Biomed. Appl. 664, 415-420 (1995).

22. Chriett, S. \& Pirola, L. Essential roles of four-carbon backbone chemicals in the control of metabolism. World J. Biol. Chem. 6, 223-230 (2015).

23. Cavaleri, F. \& Bashar, E. Potential synergies of beta-hydroxybutyrate and butyrate on the modulation of metabolism, inflammation, cognition, and general health. J. Nutr. Metab. 2018, 7195760 (2018).

24. Newman, J. C. \& Verdin, E. Beta-hydroxybutyrate: A signaling metabolite. Annu. Rev. Nutr. 37, 51-76 (2017).

25. Shimazu, T. et al. Suppression of oxidative stress by beta-hydroxybutyrate, an endogenous histone deacetylase inhibitor. Science 339, 211-214 (2013).

26. Coster, G. \& Goldberg, M. The cellular response to DNA damage: A focus on MDC1 and its interacting proteins. Nucleus 1, 166-178 (2010).

27. Włodarczyk, M. \& Nowicka, G. Obesity, DNA damage, and development of obesity-related diseases. Int. J. Mol. Sci. 20, 1146 (2019).

28. Telenga, E. D. et al. Obesity in asthma: More neutrophilic inflammation as a possible explanation for a reduced treatment response. Allergy 67, 1060-1068 (2012).

29. Conus, S., Bruno, A. \& Simon, H. U. Leptin is an eosinophil survival factor. J. Allergy Clin. Immunol. 116, 1228-1234 (2005).

30. Fritsche, K. L. The science of fatty acids and inflammation. Adv. Nutr. 6, 293S-301S (2015).

31. Alwarith, J. et al. The role of nutrition in asthma prevention and treatment. Nutr. Rev. 78, 928-938 (2020).

32. Tanigai, T. et al. Docosahexaenoic acid exerts anti-inflammatory action on human eosinophils through peroxisome proliferatoractivated receptor-independent mechanisms. Int. Arch. Allergy Immunol. 158, 375-386 (2012).

33. Li, Y. et al. Molecular recognition of nitrated fatty acids by PPAR gamma. Nat. Struct. Mol. Biol. 15, 865-867 (2008).

34. Belvisi, M. G. \& Hele, D. J. Peroxisome proliferator-activated receptors as novel targets in lung disease. Chest 134, 152-157 (2008).

35. Kabata, H., Moro, K. \& Koyasu, S. The group 2 innate lymphoid cell (ILC2) regulatory network and its underlying mechanisms. Immunol. Rev. 286, 37-52 (2018).

36. Sunadome, H. et al. Similar distribution of peripheral blood eosinophil counts in European and East Asian populations from investigations of large-scale general population studies: The Nagahama Study. Eur. Respir. J. 57, 2004101 (2021).

37. Merimee, A. J., Misbin, R. I. \& Pulkkinen, A. J. Sex variations in free fatty acids and ketones during fasting: Evidence for a role of glucagon. JCE \& $M$ 46, 414-419 (1978).

38. Ubhi, B. K. et al. Metabolic profiling detects biomarkers of protein degradation in COPD patients. Eur. Respir. J. 40, 345-355 (2012).

39. Simon, J. A., Fong, J., Bernert, J. T. J. \& Browner, W. S. Relation of smoking and alcohol consumption to serum fatty acids. Am. J. Epidemiol. 144, 325-334 (1996).

40. Wang, B. et al. A Newfound association between MDC1 functional polymorphism and lung cancer risk in Chinese. PLoS ONE 9 , e106794 (2014).

41. Lou, Z. \& Chen, J. Use of siRNA to study the function of MDC1 in DNA damage responses. Methods Mol. Biol. 281, 179-187 (2004).

42. Salati, S. et al. Eosinophils, but not neutrophils, exhibit an efficient DNA repair machinery and high nucleolar activity. Haematologica 92, 1311-1318 (2007).

43. Mizuta, K., Sasaki, H., Zhang, Y., Matoba, A. \& Emala, C. W. Sr. The short-chain free fatty acid receptor FFAR3 is expressed and potentiates contraction in human airway smooth muscle. Am. J. Physiol. Lung Cell Mol. Physiol. 318, L1248-L1260 (2020).

44. Kimura, I. et al. Short-chain fatty acids and ketones directly regulate sympathetic nervous system via G protein-coupled receptor 41 (GPR41). Proc. Natl. Acad. Sci. USA 108, 8030-8035 (2011).

45. Izuhara, Y. et al. Mouth breathing, another risk factor for asthma: the Nagahama Study. Allergy 71, 1031-1036 (2016).

46. Matsumoto, H. et al. Risks and cough-aggravating factors in prolonged cough. Epidemiological observations from the Nagahama Cohort Study. Ann. Am. Thorac. Soc. 14, 698-705 (2017).

47. Brial, F. et al. The natural metabolite 4-cresol improves glucose homeostasis and enhances beta-cell function. Cell Rep. 30, 23062320.e2305 (2020).

48. Chong, J., Wishart, D. S. \& Xia, J. Using MetaboAnalyst 4.0 for comprehensive and integrative metabolomics data analysis. Curr. Protocols Bioinformat. 68, e86 (2019).

\section{Acknowledgements}

We are incredibly grateful to the Nagahama City Office and nonprofit organization Zeroji Club for their help in conducting the Nagahama Study. 


\title{
Author contributions
}

K.N. analyzed and interpreted the data and wrote the draft. H.M. conceived and designed the study, collected, analyzed and interpreted the data, wrote and edited the manuscript. N.T., S.T., N.N., M.K., and C.M. analyzed the data. H.S., T.N., T.O., Y.N., and K.M. collected the data and revised the work critically. T.K., Y.T., K.S., F.M. contributed to the design of the Nagahama cohort study, recruited subjects, acquired the funding, and critically revised the manuscript. K.C. and T.H. provided overall supervision and critically revised the manuscript.

\section{Funding}

This study was supported by a University Grant, the Center of Innovation Program, the Global University Project, and Grant-in-Aid for Scientific Research from the Ministry of Education, Culture, Sports, Science and Technology in Japan, by the Practical Research Project for Rare/Intractable Diseases, the Comprehensive Research on Aging and Health Science Research Grants for Dementia R\&D, the Program for an Integrated Database of Clinical and Genomic Information, and the Practical Research Project for Life-Style related Diseases including Cardiovascular Diseases and Diabetes Mellitus, from Japan Agency for Medical Research and Development (AMED), and by the Takeda Medical Research Foundation.

\section{Competing interests}

The authors declare no competing interests.

\section{Additional information}

Supplementary Information The online version contains supplementary material available at https://doi.org/ 10.1038/s41598-021-94835-9.

Correspondence and requests for materials should be addressed to H.M.

Reprints and permissions information is available at www.nature.com/reprints.

Publisher's note Springer Nature remains neutral with regard to jurisdictional claims in published maps and institutional affiliations.

\begin{abstract}
Open Access This article is licensed under a Creative Commons Attribution 4.0 International License, which permits use, sharing, adaptation, distribution and reproduction in any medium or format, as long as you give appropriate credit to the original author(s) and the source, provide a link to the Creative Commons licence, and indicate if changes were made. The images or other third party material in this article are included in the article's Creative Commons licence, unless indicated otherwise in a credit line to the material. If material is not included in the article's Creative Commons licence and your intended use is not permitted by statutory regulation or exceeds the permitted use, you will need to obtain permission directly from the copyright holder. To view a copy of this licence, visit http://creativecommons.org/licenses/by/4.0/.
\end{abstract}

(C) The Author(s) 2021 